\title{
Tracking control with adaption of kites*
}

\author{
J. H. Baayen ${ }^{\dagger}$ and W. J. Ockels ${ }^{\ddagger}$
}

June 27, 2011

\begin{abstract}
A novel tracking paradigm for flying geometric trajectories using tethered kites is presented. It is shown how the differential-geometric notion of turning angle can be used as a one-dimensional representation of the kite trajectory, and how this leads to a single-input single-output (SISO) tracking problem. Based on this principle a Lyapunov-based nonlinear adaptive controller is developed that only needs control derivatives of the kite aerodynamic model. The resulting controller is validated using simulations with a point-mass kite model.
\end{abstract}

\section{Introduction}

In 1980, Loyd wrote a seminal paper exploring the possibility of generating electrical power using the pulling force of tethered airfoils, i.e., kites [19]. During the oil glut of the 1980s, however, interest in wind energy dropped [1]. It was only during the turn of the $21^{\text {st }}$ century that interest in kite power (and traction) picked up again with the work of Meijaard, Ockels, Schwab and Diehl [20, 21, 8].

Currently, almost the whole wind energy market makes use of horizontal axis wind turbines. Wind turbines harvest the energy relatively close to the ground. But realizing that up to a height of approximately 10 kilometres the wind velocity increases with altitude [18], and that the wind power raises with the wind speed to the third power [19], one concludes that there is a large potential to extract wind energy at higher altitudes. A research group at the Institute for Applied Sustainable Science, Engineering and Technology (ASSET) at Delft University of Technology is investigating a wind energy converter using kites as lifting surfaces, i.e., the Laddermill [21].

The design of automatic controllers for kite power systems has been a subject of ongoing research $[25,26,15,12,13,9,7]$. In this paper we discuss two contributions. First, we illustrate a trajectory tracking concept based on the

\footnotetext{
* This paper is a preprint of a paper submitted to IET Control Theory \& Applications and is subject to Institution of Engineering and Technology Copyright. If accepted, the copy of record will be available at IET Digital Library.

${ }^{\dagger}$ Independent Consultant, Weserstraße 153, 12045 Berlin, Germany, jorn.baayen@gmail.com. Formerly at Delft Institute of Applied Mathematics, Faculty of Electrical Engineering, Mathematics and Computer Science, Mekelweg 4, 2628 CD, Delft, The Netherlands.

‡ASSET Institute, Kluyverweg 1, 2629 HS, Delft, The Netherlands, w.j.ockels@tudelft.nl.
} 
angle between the velocity vector and the horizontal. This concept is restricted to the motion of the kite in directions perpendicular to the vector connecting the earth tether attachment point and the kite, leaving control of the reeling in and reeling out of the tether up to a separate controller. To get a first intuitive picture of this idea, let us for a moment project the crosswind figureeight trajectory of a kite onto the plane perpendicular to the wind vector. As a kite traces out a figure eight on this plane, the angle the velocity vector makes with the horizontal oscillates between certain minimum and maximum values; see Figures 1 and 2.

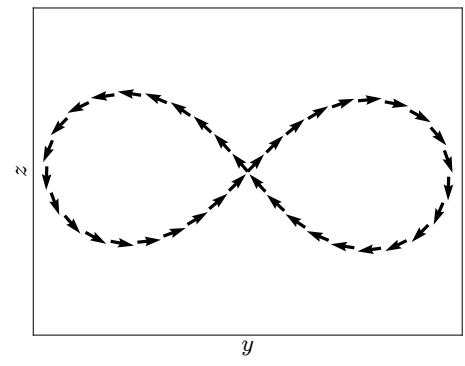

Figure 1: Tangent vectors along path.

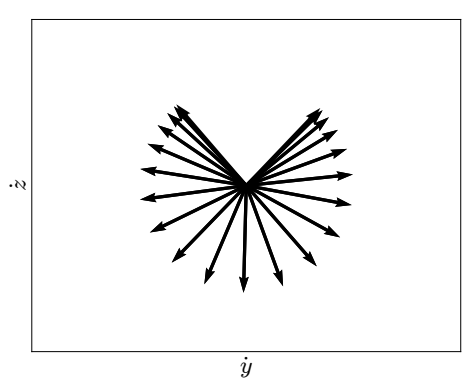

Figure 2: Tangent vectors translated to the origin.

Instead of measuring this angle directly we use the more general notion of turning angle [11] from elementary differential geometry (see the Appendices). This angle is not restricted to $[-\pi, \pi]$ and it is continuous. It is cumulative in the sense that multiple turns around the origin of the velocity vector will result in a multiple of $2 \pi$, but winding backwards will reduce the turning angle by the same amount for every turn. This property makes the turning angle well suited for control purposes. By considering the arc length as independent variable, the turning angle - together with initial conditions - becomes a representation of the image of a curve that is independent of parametrization.

Secondly, we derive an adaptive turning angle tracking controller that does not need a full aerodynamic kite model. Instead it only uses the control derivatives relating control input increments to steering force increments; the rest of the required data is measured directly. We provide a stability proof for the resulting controller and demonstrate its performance with simulations.

The remainder of this paper is organized as follows. In the next section we discuss the physics of the kite control problem and touch on previous research. In section 3 we formalize a geometric model of the dynamics of the kite trajectory, and in section 4 we formulate and solve the control problem. We illustrate the performance of the control law with simulations in section 5. Finally, the appendices list relevant results from the field of differential geometry. 


\section{Motivation}

The Laddermill uses kites to pull a tether from a drum driving a generator to produce electricity. The operational mode consists of two distinct phases: a reeling-out phase pulling the tether from the drum producing power and a reeling-in phase which consumes energy; see Figure 3. It is desired to control the kite in such a manner that a net power production remains between the reeling-out and reeling-in phase. By flying the kite in directions perpendicular to the wind, the magnitude of the airflow along the airfoil is increased [19]. This principle is comparable to the crosswind rotation of wind turbine blades.

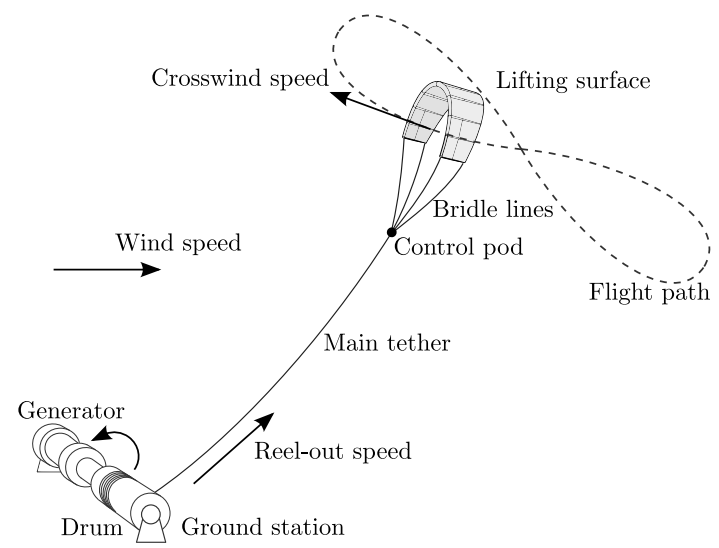

Figure 3: The Laddermill during the reel-out phase [7].

The control mechanism developed at the ASSET institute consists of a control pod suspended under the kite. The control pod is connected to the drum on the ground using a single tether and four bridle lines lead from the control pod to the kite. The pod contains two servo motors, one controlling the length difference of the two back lines, the steering lines, and the other motor controlling the overall length of the steering lines. By changing the length difference of the steering lines the kite deforms; see Figure 4. The aerodynamics in turn generate steering yaw moments. A detailed explanation is available in [5]. Regulation of the total length of the steering lines provides some control over the attack angle of the kite itself. This is known as the powering and the depowering of the kite.

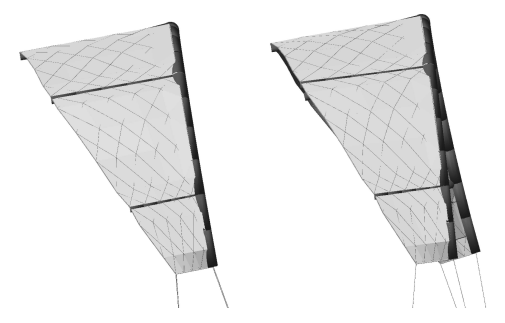

Figure 4: Kite before and after applying a steering input [5].

Previous research into automatic control of kites has focused on trajectory tracking using linearization [25, 26] and Model Predictive Control (MPC) [15, 
12, 13, 9]. All of these approaches require a fully validated kite model that can be applied in real time. Multi-body models such as those developed in [5] are precise but too computationally intensive for control design, and condensation of kite dynamics into a rigid body model using system identification techniques has proven to be difficult [7].

\section{Geometric modeling and system}

We are interested in controlling flight in directions perpendicular to that of the radial vector $\mathbf{r}$ connecting the earth tether attachment point and the kite. The control of the tether and of the power lines is left to a separate controller. In order to derive a control system that is invariant of the tether length, we project the flight trajectory onto the upper half of the unit sphere centered on the earth tether attachment point. This yields the projected path $\gamma:=\mathbf{r} /\|\mathbf{r}\|$.

\subsection{Geometric model}

As mentioned in the introduction, we will control the turning angle of the kite trajectory. In order to be able to compute the turning angle on the upper half of the unit sphere we need a coordinate system. For this, note that the coordinate patch

$$
\mathbf{p}:[0,2 \pi) \times[0, \pi / 2] \rightarrow \mathbb{R}^{3}, \quad(v, w) \mapsto(\cos w \cos v, \cos w \sin v, \sin w) .
$$

yields a continuous one-to-one (except for the pole) correspondence between the upper half of the sphere and the set $[0,2 \pi) \times[0, \pi / 2]$. The vector fields $\partial \mathbf{p} / \partial v$ and $\partial \mathbf{p} / \partial w$ are orthogonal, and by normalizing them we obtain an orthonormal basis of the tangent plane,

$$
\mathbf{e}_{1}:=(-\sin v, \cos v, 0), \quad \mathbf{e}_{2}:=(-\cos v \sin w,-\sin v \sin w, \cos w),
$$

as illustrated in Figure 12.

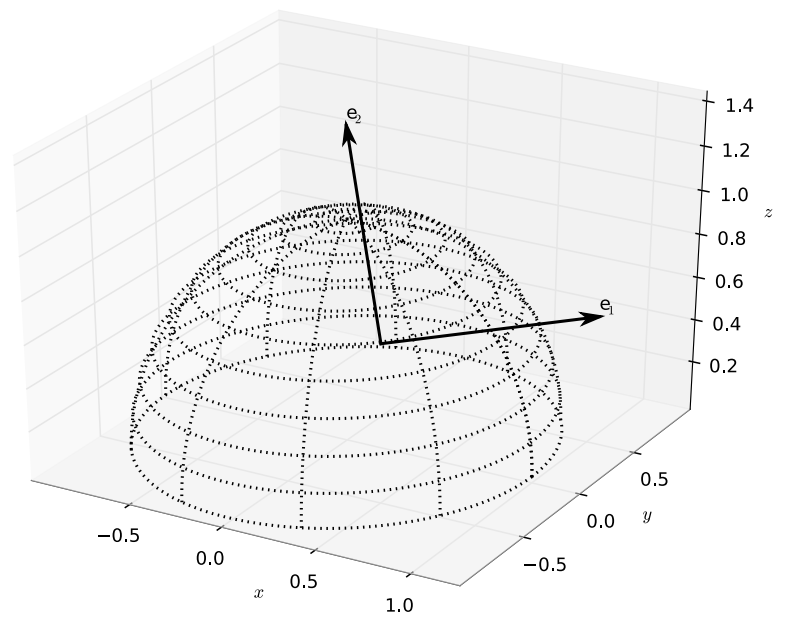

Figure 5: Coordinate system on the upper half of the unit sphere. 
The vector field $\mathbf{e}_{1}$ has no vertical component, which intuitively makes it a suitable reference for the definition of a turning angle. To make this precise, we apply Liouville's theorem [11] for which we need the curvatures of the coordinate system. The coordinate curves $w \mapsto \mathbf{p}(v, w)$ are parts of great circles, whence their geodesic curvature $\left(\kappa_{g}\right)_{2}$ is zero. The curves $v \mapsto \mathbf{p}(v, w)$, however, have geodesic curvature $\left(\kappa_{g}\right)_{1}=\sin w$. By Liouville's theorem, the turning angle of the projected kite trajectory with respect to the vector field $\mathbf{e}_{1}$ is given by the integral equation

$$
\theta[\gamma]:=\theta\left[\boldsymbol{\gamma}, \mathbf{e}_{1}\right]=\int_{\boldsymbol{\gamma}} \kappa_{g}[\boldsymbol{\gamma}]-\left(\kappa_{g}\right)_{1} \cos \theta\left[\boldsymbol{\gamma}, \mathbf{e}_{1}\right] d s+\theta\left[\boldsymbol{\gamma}, \mathbf{e}_{1}\right]_{0}
$$

where $\kappa_{g}[\gamma]$ is the geodesic curvature of the projected kite trajectory and $\theta\left[\gamma, \mathbf{e}_{1}\right]_{0}$ is the initial value of the turning angle. The geodesic curvature of the projected kite trajectory can be calculated as

$$
\kappa_{g}[\gamma]=\frac{\dot{x} \ddot{y}-\ddot{x} \dot{y}}{\|\dot{\gamma}\|^{3}}
$$

with $\dot{x}$ and $\dot{y}$ the coordinates of the velocity of the projected trajectory, expressed in the $\left(\mathbf{e}_{1}, \mathbf{e}_{2}\right)$-basis of the tangent plane.

\subsection{Path length}

The projected velocity of the kite may sometimes have a component perpendicular to the target trajectory $\gamma_{t}$. If we zig-zag around the target trajectory and straighten out the traversed path we obtain a longer arc length than if we would straighten out the target trajectory. We need to take this difference into account, for otherwise we would move through the target trajectory quicker than intended. For this reason we introduce a corrected path length that disregards the perpendicular component.

Let $\mathbf{T}_{t}:=\cos \theta\left[\boldsymbol{\gamma}_{t}\right] \mathbf{e}_{1}+\sin \theta\left[\boldsymbol{\gamma}_{t}\right] \mathbf{e}_{2}$, where $\left(\mathbf{e}_{1}, \mathbf{e}_{2}\right)$ is the orthonormal basis of the tangent plane at $\gamma_{t}$ given by (1). This unit vector field is tangent to the target trajectory. We transport this tangent vector to the tangent space at $\gamma$ by means of the operator $\mathrm{R}$, which rotates $\gamma$ to $\gamma_{t}$ along their connecting geodesic. Taking the inner product $\dot{\gamma} \cdot\left(\mathrm{R}^{T} \mathbf{T}_{t}\right)$, we obtain the component of the projected velocity tangential to the target trajectory. This motivates the following definition.

Definition 3.1. We refer to the integral

$$
s_{c}:=\int \dot{\gamma} \cdot\left(\mathrm{R}^{T} \mathbf{T}_{t}\right) d t
$$

as the corrected path length.

\subsection{Control system}

Our objective is to track the given target trajectory $\gamma_{t}$ which, as hinted at previously, we will do by controlling the turning angle $\theta[\gamma]$. We show that we can achieve this objective using a single steering control input. This way we construct a one-dimensional single-input, single-output tracking problem. 
In order to arrive at a set of equations that are affine in their control input we base our discussion on steering input increments $\tilde{u}:=u-u^{*}$. Central to this linearizing approach is the following assumption.

Assumption 3.1. The acceleration $\ddot{\gamma}$ is locally affine in feasible ${ }^{1}$ control increments $\tilde{u}$. That is,

$$
\left|\frac{\partial^{3} \gamma}{\partial u \partial t^{2}}\right| \gg\left|\frac{\partial^{4} \gamma}{\partial u^{2} \partial t^{2}} \tilde{u}\right|
$$

Linearizing the derivative of the turning angle in the control input $u$ we find, using (2), (3), and the definition of the corrected path length, that

$$
\begin{aligned}
\frac{d \theta[\gamma]}{d t} & =\left.\frac{d \theta[\gamma]}{d t}\right|_{u^{*}}+\left.\frac{\partial^{2} \theta[\gamma]}{\partial u \partial t}\right|_{u^{*}} \tilde{u} \\
& =\left.\frac{d \theta[\gamma]}{d s} \frac{d s}{d t}\right|_{u^{*}}+\left.\frac{\partial^{2} \theta[\gamma]}{\partial u \partial s} \frac{d s}{d t}\right|_{u^{*}} \tilde{u} \\
& =\frac{\dot{x} \ddot{y}-\ddot{x} \dot{y}}{\|\dot{\gamma}\|^{2}}-\gamma \cdot \mathbf{e}_{z} \cos \theta[\gamma]\|\dot{\gamma}\|+\frac{\dot{x} \frac{\partial \ddot{y}}{\partial u}-\frac{\partial \ddot{x}}{\partial u} \dot{y}}{\|\dot{\gamma}\|^{2}} \tilde{u}
\end{aligned}
$$

where $\boldsymbol{\gamma} \cdot \mathbf{e}_{z}$ denotes the (earth) vertical component of the projected trajectory. In deriving our control loop in the next section we will make use of the direct coupling between the control increment $\tilde{u}$ and the derivative of the turning angle. This principle is similar to the incremental nonlinear dynamic inversion approach for control of aircraft as developed by Sieberling et al. [23].

For the derivatives $\partial \ddot{x} / \partial u$ and $\partial \ddot{y} / \partial u$, representing the sensitivities of the accelerations to changes in the steering input, we need a model. Let $r=\|\mathbf{r}\|$ and note that

$$
\ddot{\gamma}=\frac{d^{2}}{d t^{2}}\left(\frac{\mathbf{r}}{r}\right)=\frac{\ddot{\mathbf{r}}}{r}-\frac{\ddot{r} \mathbf{r}}{r^{2}}-2 \frac{\dot{r} \mathbf{r}}{r^{2}}+2 \frac{\dot{r}^{2} \mathbf{r}}{r^{3}} .
$$

Expressed in the tangential frame the first two coordinates of the radial vector $\mathbf{r}$ are zero. As a result, and due to the fact that only the control derivatives of the accelerations are non-zero, we see that the sensitivities, expressed in the tangential frame, are given by

$$
\begin{aligned}
& \frac{\partial \ddot{x}}{\partial u}=\frac{\rho v_{a}^{2} S_{t} C_{x, u}^{t}}{2 m r}, \\
& \frac{\partial \ddot{y}}{\partial u}=\frac{\rho v_{a}^{2} S_{t} C_{y, u}^{t}}{2 m r} .
\end{aligned}
$$

Here $C_{x, u}^{t}$ and $C_{y, u}^{t}$ are the unitless control derivatives expressed in the tangential frame, $\rho$ is the density of air, $v_{a}$ is the airspeed, $S_{t}$ is the surface area of the tips and $m$ is the combined mass of the kite and the control pod. Let $\mathbf{C}_{u}=\left[\begin{array}{llll}C_{x, u} & C_{y, u} & C_{z, u}\end{array}\right]$ denote the control derivatives expressed in a body-fixed reference frame.

Kites deform during flight and at any point in time the deformation depends on the form the kite had previously. Hence the deformation of the kite is pathdependent. In order to model the control derivatives, we assume path-dependent deformation to have a - for control purposes - negligible influence on the steering aerodynamics.

\footnotetext{
${ }^{1}$ We call a control input increment feasible when the actuator can implement it within a single sampling interval.
} 
Assumption 3.2. The unitless control derivatives $\mathbf{C}_{u}$, expressed in the bodyfixed reference frame, are a function of the current steering input $u$.

Note that this assumption can be relaxed by including the dependence on additional state variables.

We approximate the function $u \mapsto \mathbf{C}_{u}$, mapping states to control derivatives, using B-spline networks as in [24]. Let $\mathbf{w}_{i}$ denote the weights and $\mathbf{b}$ the basis functions of the B-spline network for the $i$ th control derivative with $i \in\{x, y, z\}$, so that the $i$ th B-spline network is given by $C_{i, u}=\mathbf{w}_{i} \cdot \mathbf{b}$. The number of basis functions used for the spline networks must be chosen in such a way as to avoid problems with under- or overparametrization. This can be done using a trial and error procedure.

By continuously measuring the position, linear velocity, linear acceleration, airspeed and attitude of the kite, we obtain a nonlinear system of the form

$$
\frac{d \theta[\gamma]}{d t}=f(\theta[\gamma], t)+B(t) \tilde{u}
$$

The measurements are implicit in the time-dependence of the functions $f$ and $B$, which are given by

$$
f=\left.\frac{d \theta[\gamma]}{d t}\right|_{u^{*}}=\frac{\dot{x} \ddot{y}-\ddot{x} \dot{y}}{\|\dot{\gamma}\|^{2}}-\gamma \cdot \mathbf{e}_{z} \cos \theta[\gamma]\|\dot{\gamma}\|
$$

and

$$
B=\left.\frac{\partial^{2} \theta[\gamma]}{\partial u \partial t}\right|_{u^{*}}=\frac{\dot{x} \frac{\partial \ddot{y}}{\partial u}-\frac{\partial \ddot{x}}{\partial u} \dot{y}}{\|\dot{\gamma}\|^{2}} .
$$

By choosing our system in this way the need for a full aerodynamic kite model vanishes.

From (4) and (5) we see that the function $B$ is linear in the control derivatives $C_{i, u}=\mathbf{w}_{i} \cdot \mathbf{b}$, that is,

$$
B=\sum_{i} \lambda_{i} \mathbf{w}_{i} \cdot \mathbf{b}
$$

for some coefficients $\lambda_{i} \in \mathbb{R}$. This shows that the system (6) is affine in both the control input $\tilde{u}$ as well as in the control derivative spline weights $\mathbf{w}_{i}$, a property that will allow us to design an adaptive control loop with relative ease in the next section.

\section{Control}

In this section we design our control loop and control derivative estimators by means of a control-Lyapunov approach. Our approach is closely related to those described in $[24,3,16,17,14]$.

Our objective is to track a given reference projected kite trajectory. To this end, we construct a flight controller consisting of an inner and an outer loop. The inner loop controls the turning angle of the kite trajectory $\theta[\gamma]$ towards a target turning angle $\theta_{t}$. The outer loop computes a target turning angle $\theta_{t}$ that steers the kite onto the target trajectory. 


\subsection{Inner loop}

Our inner loop cost function is the difference between the actual and desired turning angle:

$$
e:=\theta[\gamma]-\theta_{t}
$$

In order to track the reference trajectory we must find a control law that renders $e \rightarrow 0$.

The steering dynamics of a kite may subtly change over time due to weather patterns and wear. In order to automatically adapt to these changes we aim for an adaptive control approach that maintains and updates an internal representation of the steering control derivatives. We will denote this internal representation as $\hat{\mathbf{w}}_{i}$ with $i \in\{x, y, z\}$.

Summarizing, we formulate the following control problem:

Problem 4.1. Find a steering control law and a control derivative B-spline weight estimator update laws that such that $e \rightarrow 0$ as $t \rightarrow \infty$.

\subsubsection{Control design}

In this section we propose steering control and estimator update laws to solve Problem 4.1 using a control-Lyapunov approach.

Proposition 4.1. Consider the system (6). The control law

$$
\tilde{u}:=-\frac{1}{\sum_{i} \lambda_{i} \hat{\mathbf{w}}_{i} \cdot \mathbf{b}}\left[\left.\frac{d \theta[\gamma]}{d t}\right|_{u^{*}}-\frac{d \theta_{t}}{d t}+K e\right],
$$

with gain $K \in \mathbb{R}^{+}$, and the control derivative $B$-spline weight estimator update laws

$$
\frac{d \hat{\mathbf{w}}_{i}}{d t}:=\Gamma e \lambda_{i} \tilde{u} \mathbf{b}
$$

with adaptation gain $\Gamma \in \mathbb{R}^{+}$, render the Lyapunov function

$$
V:=\frac{1}{2} e^{2}+\frac{1}{2} \Gamma^{-1} \sum_{i}\left\|\hat{\mathbf{w}}_{i}-\mathbf{w}_{i}\right\|^{2}
$$

decreasing along the flow.

Proof. The time-derivative of the Lyapunov function $V$ is given by

$$
\frac{d V}{d t}=e \frac{d e}{d t}+\Gamma^{-1} \sum_{i}\left(\hat{\mathbf{w}}_{i}-\mathbf{w}_{i}\right) \cdot \frac{d \hat{\mathbf{w}}_{i}}{d t}
$$

with, using (4),

$$
\frac{d e}{d t}=\left.\frac{d \theta[\gamma]}{d t}\right|_{u^{*}}+\left.\frac{\partial^{2} \theta[\gamma]}{\partial u \partial t}\right|_{u^{*}} \tilde{u}-\frac{d \theta_{t}}{d t} .
$$

The estimator update laws (11) cancel out all terms with $\mathbf{w}_{i}$. Indeed, replacing these into (13), we obtain, using (7) and (8), that

$$
\frac{d V}{d t}=e\left[\left.\frac{d \theta[\gamma]}{d t}\right|_{u^{*}}-\frac{d \theta_{t}}{d t}\right]+e \tilde{u} \sum_{i} \lambda_{i} \hat{\mathbf{w}}_{i} \cdot \mathbf{b}
$$


Finally, substituting the control law (10) into (14), we see that

$$
\frac{d V}{d t}=-K e^{2}
$$

The gain $K \in \mathbb{R}^{+}$renders $V$ strictly decreasing whenever $e \neq 0$.

In the previous analysis we neglected rate and magnitude constraints of the control signal. Let $\tilde{u}_{a}$ denote the control increment implemented by the actuator. Since in general $\tilde{u}_{a} \neq \tilde{u}$, the Lyapunov function (12) is no longer guaranteed to be decreasing and the parameter estimation process may diverge. In order to remedy this situation we introduce the modified tracking error as in [10],

$$
e_{m}:=e-\zeta
$$

with

$$
\frac{d \zeta}{d t}:=-K \zeta+\left.\frac{\partial^{2} \theta[\gamma]}{\partial u \partial t}\right|_{u^{*}, \hat{\mathbf{w}}_{i}}\left(\tilde{u}_{a}-\tilde{u}\right),
$$

providing a correction for the influence of the actuator limitations, where the second derivative is evaluated using the estimated weights $\hat{\mathbf{w}}_{i}$. Note that in the absence of actuator constraints $e_{m} \rightarrow e$.

In order to render the modified tracking error to zero, we adjust the estimator update laws (11) to be proportional to $e_{m}$. The associated modified Lyapunov function is - by construction - decreasing along the flow, independently of whether or not $\tilde{u}_{a}=\tilde{u}$ :

Proposition 4.2. Consider the system (6). Any control law and the modified control derivative B-spline weight estimator update laws

$$
\frac{d \hat{\mathbf{w}}_{i}}{d t}:=\Gamma e_{m} \lambda_{i} \tilde{u}_{a} \mathbf{b}
$$

render the modified Lyapunov function

$$
V_{m}:=\frac{1}{2} e_{m}^{2}+\frac{1}{2} \Gamma^{-1} \sum_{i}\left\|\hat{\mathbf{w}}_{i}-\mathbf{w}_{i}\right\|^{2}
$$

decreasing along the flow.

Proof. The time-derivative of the modified Lyapunov function (17) is given by

$$
\frac{d V_{m}}{d t}=e_{m} \frac{d e_{m}}{d t}+\Gamma^{-1} \sum_{i}\left(\hat{\mathbf{w}}_{i}-\mathbf{w}_{i}\right) \cdot \frac{d \hat{\mathbf{w}}_{i}}{d t}
$$

with, using (15) and (4),

$$
\frac{d e_{m}}{d t}=K\left(e-e_{m}\right)-\left.\frac{\partial^{2} \theta[\gamma]}{\partial u \partial t}\right|_{u^{*}, \hat{\mathbf{w}}_{i}}\left(\tilde{u}_{a}-\tilde{u}\right)+\left.\frac{d \theta[\boldsymbol{\gamma}]}{d t}\right|_{u^{*}}+\left.\frac{\partial^{2} \theta[\boldsymbol{\gamma}]}{\partial u \partial t}\right|_{u^{*}} \tilde{u}_{a}-\frac{d \theta_{t}}{d t} .
$$

The modified estimator update laws (16) cancel out all terms with $\mathbf{w}_{i}$. Indeed, replacing these into (18) we obtain, using (7) and (8), that

$$
\begin{aligned}
\frac{d V_{m}}{d t}=e_{m} & {\left[K\left(e-e_{m}\right)-\left.\frac{\partial^{2} \theta[\boldsymbol{\gamma}]}{\partial u \partial t}\right|_{u^{*}, \hat{\mathbf{w}}_{i}}\left(\tilde{u}_{a}-\tilde{u}\right)\right] } \\
+ & e_{m}\left[\left.\frac{d \theta[\boldsymbol{\gamma}]}{d t}\right|_{u^{*}}-\frac{d \theta_{t}}{d t}\right]+e_{m} \tilde{u}_{a} \sum_{i} \lambda_{i} \hat{\mathbf{w}}_{i} \cdot \mathbf{b} .
\end{aligned}
$$


Noting that $\tilde{u}_{a}=\left(\tilde{u}_{a}-\tilde{u}\right)+\tilde{u}$ and substituting the canonical control law (10) into (19) we see that

$$
\frac{d V_{m}}{d t}=-K e_{m}^{2} .
$$

The gain $K \in \mathbb{R}^{+}$renders $V_{m}$ strictly decreasing whenever $e_{m} \neq 0$.

\subsubsection{Control performance}

In this section we investigate the performance of the proposed control and estimator update laws. In the case that $\tilde{u}_{a}=\tilde{u}$, the function $V$ defined in (12) is decreasing along the flow. This implies convergence of the kite trajectory turning angle $\theta[\gamma]$ to the target turning angle $\theta_{t}$.

Theorem 4.1. Consider the system (6), the control law (10) and the estimator update laws (16). Assume that there is a $t_{0}>0$ such that for all $t>t_{0}$

$$
\|\dot{\gamma}(t)\|>0
$$

holds, so that for $t>t_{0}$ the kite trajectory is a regular curve. Additionally assume that for all $t>t_{0}$ the geometric condition

$$
\left.\frac{d \ddot{\gamma}}{d u}\right|_{t} \nvdash \mathbf{r}(t)
$$

i.e. that the given two vectors are not parallel, is satisfied, so that the control law is well-defined. Finally, assume that for some $M \in \mathbb{R}^{+}$and for all $t>0$ the bounds

$$
\left.\left|\frac{d \theta[\boldsymbol{\gamma}]}{d t}\right|_{t}|\leq M, \quad| \frac{d \theta_{t}}{d t}\right|_{t}|\leq M, \quad| \theta[\gamma](t)-\theta_{t}(t) \mid \leq M
$$

hold. Then, for any initial condition and for any reference trajectory the turning angle of the kite trajectory converges to the target:

$$
\lim _{t \rightarrow \infty} \theta[\gamma](t)=\theta_{t}(t)
$$

Proof. Barbalat's Lemma [16] applied to the Lyapunov function $V$ shows that $e \rightarrow 0$.

We proceed to show that, even when $\tilde{u}_{a} \neq \tilde{u}$, the modified parameter estimation process is stable in the sense that the weight estimation errors remain bounded:

Theorem 4.2. Consider the system (6) and the estimator update laws (16). Assume that there is a $t_{0}>0$ such that for all $t>t_{0}$ the conditions

$$
\|\dot{\gamma}(t)\|>0,
$$

and

$$
\left.\frac{d \ddot{\gamma}}{d u}\right|_{t} \nVdash \mathbf{r}(t)
$$

are satisfied. Then for any initial estimates, any reference trajectory and any actuated control increments $\tilde{u}_{a}$, the estimator errors

$$
\left\|\hat{\mathbf{w}}_{i}(t)-\mathbf{w}_{i}(t)\right\|, \quad i \in\{x, y, z\}
$$

are bounded, and the bound decreases in time for $t>t_{0}$. 
Proof. Boundedness follows from the definition of the Lyapunov function $V_{m}$ :

$$
\left\|\hat{\mathbf{w}}_{i}(t)-\mathbf{w}_{i}(t)\right\| \leq \sqrt{2 \Gamma V_{m}(t)} .
$$

Proposition 4.2 shows that the bound decreases in time.

We do not, however, have a guarantee that the estimates will converge to the true values of the weights. The modified Lyapunov function $V_{m}$ becomes stationary once $e_{m}=0$, and at this point the estimation error will not be able to decrease further.

\subsection{Outer loop}

The task of the outer loop controller is to compute an appropriate turning angle. To this end we consider the outer loop nonlinear system of the form

$$
\frac{d \gamma}{d t}=v\left(\cos \theta_{t} \mathbf{e}_{1}+\sin \theta_{t} \mathbf{e}_{2}\right),
$$

where the velocity $v=v(t) \geq 0$ is determined by the dynamics of the kite. For this system we obtain the following control problem:

Problem 4.2. Find a turning angle control law such that $\gamma \rightarrow \gamma_{t}$ as $t \rightarrow \infty$.

\subsubsection{Control design}

In this section we propose a turning angle control law to solve Problem 4.2 using a control-Lyapunov approach.

Recall that $\mathbf{T}_{t}$ is a unit vector tangent to the target trajectory; cf Section 3.2. Let $\mathbf{T}:=\mathrm{R}^{T} \mathbf{T}_{t}$, where the operator $\mathrm{R}$ rotates $\gamma$ to $\gamma_{t}$ along their connecting geodesic, so that $\mathbf{T}$ is an element of the tangent plane at $\boldsymbol{\gamma}$. Let $\mathbf{t}:=\gamma \times \mathbf{T}$, so that the vectors $\mathbf{T}$ and $\mathbf{t}$ form a basis of the tangent plane at $\boldsymbol{\gamma}$. Finally, denote

$$
\gamma_{t}^{\perp}:=\gamma_{t}-\left(\gamma_{t} \cdot \gamma\right) \gamma
$$

Proposition 4.3. The turning angle $\theta_{t}$ determined by

$$
\cos \theta_{t} \mathbf{e}_{1}+\sin \theta_{t} \mathbf{e}_{2}=\mathbf{T}+L\left(\mathbf{t} \cdot \gamma_{t}^{\perp}\right) \mathbf{t},
$$

with gain $L \in \mathbb{R}^{+}$, renders the geodesic distance

$$
W=\operatorname{dist}\left(\boldsymbol{\gamma}, \gamma_{t}\right)
$$

decreasing along the flow.

Proof. The time-derivative of the geodesic distance is given by Lemma 6.2 in Appendix B:

$$
\frac{d W}{d t}=-\left(\dot{\gamma}-\mathrm{R}^{T} \dot{\gamma}_{t}\right) \cdot \mathbf{Y}_{\boldsymbol{\gamma}}^{\gamma_{t}}
$$

where $\mathbf{Y}_{\gamma}^{\gamma_{t}}$ is the geodesic vector pointing from $\gamma$ towards $\gamma_{t}$. Substituting (21) into (22), and noting that

$$
\dot{\gamma}_{t}=(\dot{\gamma} \cdot \mathbf{T}) \mathbf{T}_{t},
$$

we obtain

$$
\frac{d W}{d t}=-L v\left(\mathbf{t} \cdot \mathbf{Y}_{\gamma}^{\gamma_{t}}\right)\left(\mathbf{t} \cdot \gamma_{t}^{\perp}\right)=-L v \frac{\left(\mathbf{t} \cdot \boldsymbol{\gamma}_{t}^{\perp}\right)^{2}}{\left\|\gamma_{t}^{\perp}\right\|} .
$$

Note that $d W / d t \rightarrow 0$ as $\gamma_{t}^{\perp} \rightarrow 0$. 


\subsubsection{Control performance}

Proposition 4.2.1 shows that the control law (21) renders the geodesic distance $\operatorname{dist}\left(\boldsymbol{\gamma}, \boldsymbol{\gamma}_{t}\right)$ decreasing along the flow. In this sense, the control law (21) renders the target trajectory stable, but not asymptotically so. For the latter we would also need to be able to control the kite velocity $v$.

\section{Simulation}

In this section we report on the performance of our controller with an extension of a point-mass kite model commonly found in the literature.

\section{$5.1 \quad$ Model}

We present an extension of the point-mass kite model discussed in $[8,12,13$, 15, 9]. For simplicity, our presentation uses a centripetal force to keep the kite on a sphere with given radius.

We express the aerodynamic forces acting on the kite in an aerodynamic reference frame. Let $\mathbf{v}_{a}=\dot{\mathbf{r}}-\mathbf{v}_{w}$ denote the apparent wind vector, where $\mathbf{v}_{w}$ represents the environmental wind. Let $\mathbf{e}_{x}$ be the unit vector collinear with the apparent wind vector $\mathbf{v}_{a}$. We assume that the kite cannot roll with respect to the tether, and so we must have $\mathbf{e}_{y}$ such that $\mathbf{e}_{y}$ is parallel to $\mathbf{r} \times \mathbf{e}_{x}$. Choose $\mathbf{e}_{y}$ to be collinear with $\mathbf{r} \times \mathbf{e}_{x}$ and let $\mathbf{e}_{z}:=\mathbf{e}_{x} \times \mathbf{e}_{y}$.

In expressing the magnitude of the forces we will make use of unitless lift coefficients. Let $C_{L}: \alpha \mapsto C_{L}(\alpha)$ be the unitless lift curve with $\alpha:=\operatorname{acos}(\mathbf{r}$. $\left.\mathbf{e}_{z} /\|\mathbf{r}\|\right)+\theta_{p}$, where $\theta_{p}$ is the power setting, and let $C_{S}: u \mapsto C_{S}(u)$ be the unitless steering force curve. We model the drag force as the sum of a zero-lift drag coefficient and a parabolic approximation of induced drag [2]:

$$
C_{D}=C_{D_{0}}+k C_{L}^{2} .
$$

The constant $k$ is defined by $k^{-1}=\pi A e$, with aspect ratio $A=b^{2} / S$, Oswald factor $e$, projected surface area $S$, and wingspan $b$. It follows that the aerodynamic forces are given by

$$
\begin{aligned}
F_{x} & =-\frac{1}{2} \rho\left\|\mathbf{v}_{a}\right\|^{2} S C_{D}(\alpha), \\
F_{y} & =-\frac{1}{2} \rho\left\|\mathbf{v}_{a}\right\|^{2} S C_{S}(u), \\
F_{z} & =-\frac{1}{2} \rho\left\|\mathbf{v}_{a}\right\|^{2} S C_{L}(\alpha),
\end{aligned}
$$

where $\rho$ is the density of air.

In our model the steering control input $u$ causes the kite to accelerate in the direction of the $\mathrm{Y}$-axis, which in turn causes an increase in the relevant component of the apparent wind vector $\mathbf{v}_{a}$. The aerodynamic reference frame rotates along with the apparent wind vector, and in this way the effects of the steering yaw moments observed in real kites are emulated. The model is completed by considering gravity and constraint forces to restrict the kite to a sphere. 
In our simulations we choose a projected surface area of $S=11 \mathrm{~m}^{2}$, a wing span of $b=6 \mathrm{~m}$, an Oswald factor of $e=0.7$ and a mass of $m=1.5 \mathrm{~kg}$. The zero-lift drag coefficient is given by $C_{D_{0}}=0.075$. The lift curve $C_{L}$, modeled on data from [22] for a NACA 0018 airfoil in a flow with Reynolds number $2 \times 10^{6}$ using B-splines, is given in Figure 6. Due to the current lack of more specific data, we also set $C_{S}=C_{L}$. This is justified by the observation that one can view the tips of the kite as vertically positioned airfoils, with a local attack angle controlled using the steering control input $u$.

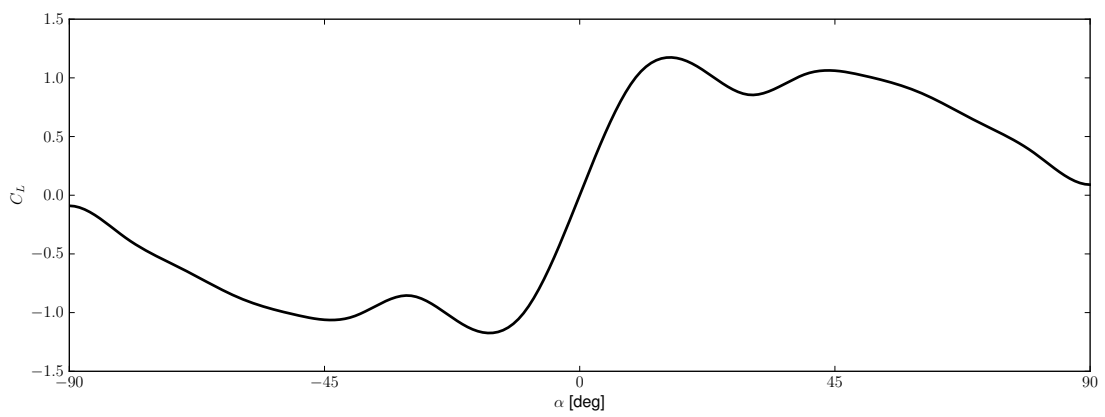

Figure 6: Lift curve modeled on data from [22] for a NACA 0018 airfoil at Re $=2 \times 10^{6}$.

\subsection{Reference trajectory}

For the tracking objective to be independent of the velocity we treat the corrected path length - as opposed to time - as the independent variable. Our reference turning angle is

$$
\theta\left[\gamma_{t}\right]\left(s_{c}\right):=A\left[\cos \left(\frac{2 \pi}{L} s_{c}\right)-1\right]+0.834029
$$

where $A \approx 2.40483$ is a root of the 0th Bessel function of the first kind and $L=4 / 3$ is the length of the projected target trajectory. This turning angle generates a figure eight trajectory. It can be shown that, for the planar notion of turning angle, the constant $A$ being a root of the appropriate Bessel function is a necessary and sufficient condition for the turning angle (23) to result in an $L$-periodic trajectory. The second constant is the initial condition which also corrects for the curvature of the sphere. It was found using a root-finding procedure. The resulting trajectory is an idealization of trajectories flown using the prototype Laddermill kite power plant.

\subsection{Results}

The wind vector points in the direction of the earth $\mathrm{X}$-axis with magnitude $6 \mathrm{~m} / \mathrm{s}$. The kite is tethered on a $100 \mathrm{~m}$ line, and the controller gains are tuned - using a trial and error procedure - to $K=3.0, L=10.0$, and $\Gamma=100.0$. The steering rate is limited to $0.05 \mathrm{rad} / \mathrm{s}$, the magnitude of the steering input to 0.12 $\mathrm{rad}$, the power setting is fixed at $\theta_{p}=0.01 \mathrm{rad}$, and every control derivative estimator B-spline has 10 knots. 
Simulation results for a run on constant line length without turbulence are plotted in Figures 7 and 8 . Part of a trajectory with a tether reel out velocity equal to a third of the wind speed is shown in Figure 10.

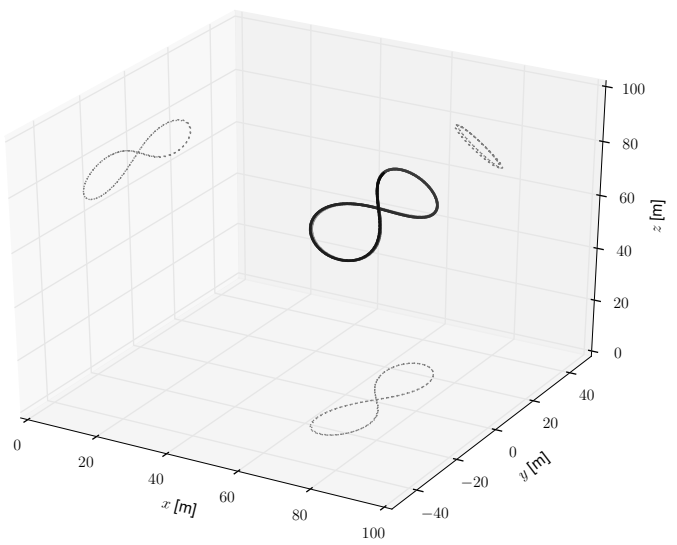

Figure 7: Three overlapping figure eight trajectories.

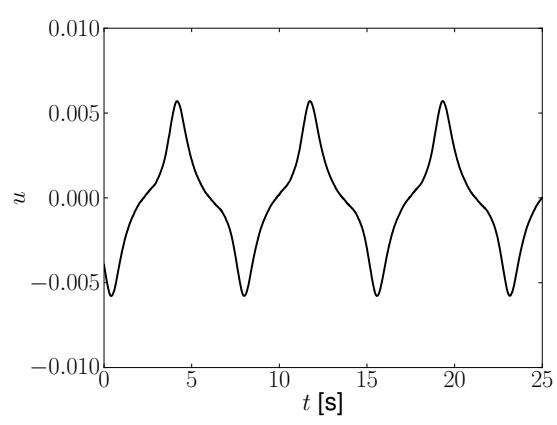

Figure 8: Control input for the trajectory shown in Figure 7.

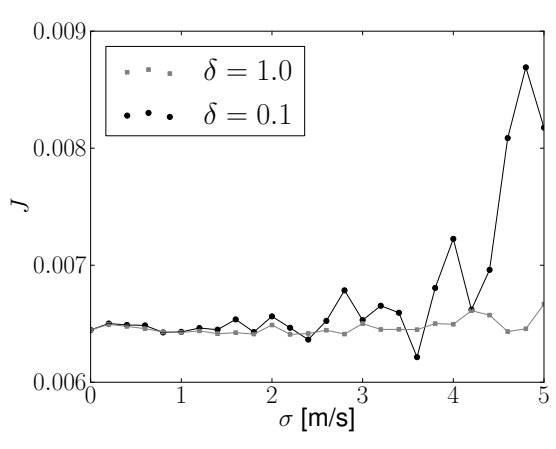

Figure 9: Mean linear tracking error as function of turbulence intensity $\sigma$ and correlation rate $\delta$, where $\sigma_{\text {lo }}=$ $\sigma_{\mathrm{la}}=\sigma_{\mathrm{ve}}=\sigma$. Every data point is an average of 100 simulations.

\subsubsection{Turbulence}

In order to quantify the controller performance under the influence of turbulence we consider the mean linear tracking error

$$
J:=\frac{1}{T} \int_{0}^{T}\left\|\gamma-\gamma_{t}\right\| d t .
$$

Figure 9 plots the mean linear tracking error $J$ for a single cycle as a function of turbulence intensity for precise initial control derivative estimates. The turbulence is modeled according to the simplified Dryden model [4]. The simulation 


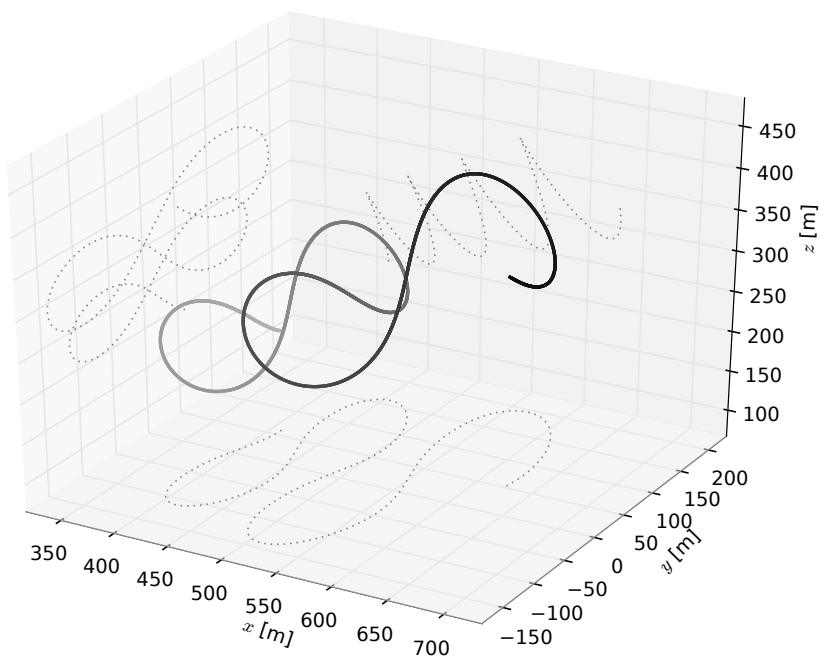

Figure 10: Part of a trajectory during tether reel out.

runs at $100 \mathrm{~Hz}$ and every data point is an average of 100 simulations. The graph shows that for the investigated range of turbulence intensities the tracking error remains bounded by a value of 0.009 . Scaling this number with the distance between the earth tether attachment point and the kite $(100 \mathrm{~m})$ we obtain a bound of less than one meter.

\subsubsection{Measurement noise}

Noisy measurements degrade control performance, which might conceivably lead to problems with adaptivity. Initial investigations suggest that measurement noise does not induce drift in the estimates of the control derivatives; see Figure 11 for the evolution of a control derivative estimate with Gaussian white noise at the levels given in Table 1. Assessment of the exact effects of measurement noise on adaptivity performance is a topic for further investigation and is beyond the scope of this work.

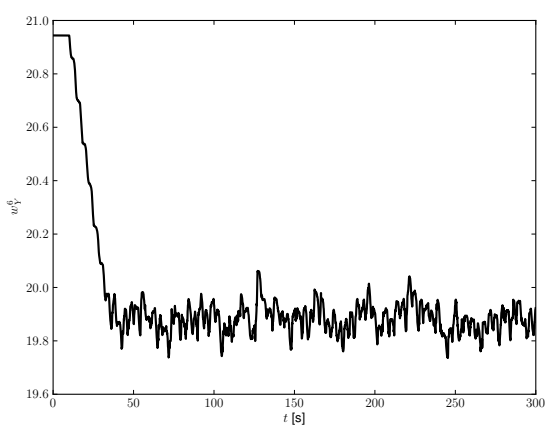

Figure 11: Evolution of the 6th Y-axis control derivative estimate with Gaussian white noise at the levels given in Table 1. 


\begin{tabular}{|l|l|l|}
\hline Measurement & Mean & Standard deviation \\
\hline Position & $0 \mathrm{~m}$ & $2.5 \mathrm{~m}$ \\
Velocity & $0 \mathrm{~m} / \mathrm{s}$ & $0.02 \mathrm{~m} / \mathrm{s}$ \\
Acceleration & $0 \mathrm{~m} / \mathrm{s}^{2}$ & $0.02 \mathrm{~m} / \mathrm{s}^{2}$ \\
Airspeed & $0 \mathrm{~m} / \mathrm{s}$ & $0.9 \mathrm{~m} / \mathrm{s}$ \\
Attitude & $0 \mathrm{deg}$ & $1 \mathrm{deg}$ \\
\hline
\end{tabular}

Table 1: Measurement noise levels.

\section{Concluding remarks}

In this paper we designed a controller for trajectory tracking control of kites. We projected the kite trajectory onto a sphere and used the differential-geometric notion of turning angle as primary tracking variable. Based on this dimension reduction we derived an adaptive controller with minimal modelling requirements. Repeated simulations with a point-mass model show our control approach to be robust against turbulence. A sequel paper will detail the performance of our controller with multi-body simulations of a deforming kite on a flexible tether.

\section{Acknowledgments}

The authors are indebted to Dr. Q. P. Chu for his excellent course on advanced flight control at Delft University of Technology, to prof. J. H. van Schuppen, Dr. R. Schmehl and Dr. D. Jeltsema for their comments and supervision, to Dipl.-Ing. M. Klaus at NTS Energie- und Transportsysteme GmbH for fruitful discussions, and to the entire Laddermill team for their support and suggestions.

\section{Appendix A: Geodesic curvature}

In this section we list the main results related to the concept of geodesic curvature referred to in this paper, adapted from the book by Gray [11]. We will not make the notions of surface and curve precise here, but only note that a coordinate patch is a differentiable map between an open set $\mathcal{U} \subset \mathbb{R}^{2}$ and $\mathbb{R}^{3}$ : the intuition is that it associates coordinates to a part of a surface.

In the following, $J$ will denote a counterclockwise rotation by $\pi / 2$ in the tangent plane of the surface under consideration.

We start with the formal definition of the turning angle. Intuitively, the turning angle is the angle between the tangent of a curve and a reference, without being restricted to $[-\pi, \pi]$ :

Definition 6.1. Let $\mathcal{M}$ be an oriented surface, suppose that $\alpha:(a, b) \rightarrow \mathcal{M}$ is a regular curve, and that $\mathbf{X}$ is an everywhere nonzero vector field along $\boldsymbol{\alpha}$. Fix $t_{0}$ with $a<t_{0}<b$. Let $\theta_{0}$ be a number such that

$$
\frac{\dot{\boldsymbol{\alpha}}\left(t_{0}\right)}{\left\|\dot{\boldsymbol{\alpha}}\left(t_{0}\right)\right\|}=\cos \theta_{0} \frac{\mathbf{X}\left(t_{0}\right)}{\left\|\mathbf{X}\left(t_{0}\right)\right\|}+\sin \theta_{0} \frac{J \mathbf{X}\left(t_{0}\right)}{\left\|\mathbf{X}\left(t_{0}\right)\right\|} .
$$


Then there exists a unique differentiable function $\theta=\theta[\boldsymbol{\alpha}, \mathbf{X}]:(a, b) \rightarrow \mathbb{R}$ such that $\theta\left(t_{0}\right)=\theta_{0}$ and

$$
\frac{\dot{\boldsymbol{\alpha}}}{\|\dot{\boldsymbol{\alpha}}\|}=\cos \theta[\boldsymbol{\alpha}, \mathbf{X}] \frac{\mathbf{X}}{\|\mathbf{X}\|}+\sin \theta[\boldsymbol{\alpha}, \mathbf{X}] \frac{J \mathbf{X}}{\|\mathbf{X}\|},
$$

at all points on the curve. We call $\theta[\boldsymbol{\alpha}, \mathbf{X}]$ the turning angle of $\boldsymbol{\alpha}$ with respect to $\mathbf{X}$ determined by $\theta_{0}$ and $t_{0}$.

In order to state Liouville's theorem, which allows us to compute the turning angle of a curve, we need the notion of geodesic curvature. Intuitively speaking the geodesic curvature of a curve measures how far it is from being a geodesic, that is, the shortest path between two points on the surface:

Definition 6.2. Let $\boldsymbol{\alpha}:(a, b) \rightarrow \mathcal{M}$ be a curve in an oriented regular surface $\mathcal{M}$ in $\mathbb{R}^{3}$. Then the geodesic curvature of $\boldsymbol{\alpha}$ is defined (for $a<t<b$ ) as

$$
\kappa_{g}[\boldsymbol{\alpha}](t)=\frac{\ddot{\boldsymbol{\alpha}}(t) \cdot J \dot{\boldsymbol{\alpha}}(t)}{\|\dot{\boldsymbol{\alpha}}(t)\|^{3}} .
$$

Finally, we state Liouville's theorem, which relates the geodesic curvature of a curve to the geodesic curvatures of the coordinate lines induced by the coordinate patch and the turning angle of the curve. Let $\left(\kappa_{g}\right)_{1}$ and $\left(\kappa_{g}\right)_{2}$ denote the geodesic curvatures of the coordinate lines $u \mapsto \mathbf{x}(u, v)$ and $v \mapsto \mathbf{x}(u, v)$, where $\mathbf{x}$ is a given patch.

Theorem 6.1 (Liouville). Let $\mathcal{M}$ be an oriented surface, and suppose that $\boldsymbol{\alpha}:(a, b) \rightarrow \mathcal{M}$ is a regular curve whose trace is contained in $\mathbf{x}(\mathcal{U})$, where $\mathbf{x}: \mathcal{U} \rightarrow \mathcal{M}$ is a coordinate patch such that $\mathbf{x}_{v}=J \mathbf{x}_{u}$. Then

$$
\kappa_{g}[\boldsymbol{\alpha}]=\left(\kappa_{g}\right)_{1} \cos \theta+\left(\kappa_{g}\right)_{2} \sin \theta+\frac{\dot{\theta}}{\|\dot{\boldsymbol{\alpha}}\|},
$$

where $\theta=\theta\left[\boldsymbol{\alpha}, \mathbf{x}_{u}\right]$ is the angle between $\boldsymbol{\alpha}$ and $\mathbf{x}_{u}$.

\section{Appendix B: Geodesic distance on the sphere}

In this section we list the main results related to the concept of geodesic distance on the sphere referred to in this paper, adapted from the report by Bullo, Murray, and Sarti [6].

Definition 6.3. The geodesic distance between the points $\mathbf{p}$ and $\mathbf{q}$ on the unit sphere is defined as

$$
\operatorname{dist}(\mathbf{p}, \mathbf{q}):=\operatorname{acos} \mathbf{p} \cdot \mathbf{q} .
$$

Additionally, provided that $\mathbf{p} \neq \pm \mathbf{q}$, there is a uniquely defined unit vector that gives the geodesic direction at the point $\mathbf{p}$ towards the point $\mathbf{q}$ :

Definition 6.4. The geodesic vector giving the geodesic direction in a point $p$ towards a point $q \neq p$ is defined as

$$
\mathbf{Y}_{\mathbf{p}}^{\mathbf{q}}:=\frac{(\mathbf{p} \times \mathbf{q}) \times \mathbf{p}}{\|(\mathbf{p} \times \mathbf{q}) \times \mathbf{p}\|}=\frac{\mathbf{q}-(\mathbf{q} \cdot \mathbf{p}) \mathbf{p}}{\|\mathbf{q}-(\mathbf{q} \cdot \mathbf{p}) \mathbf{p}\|} .
$$




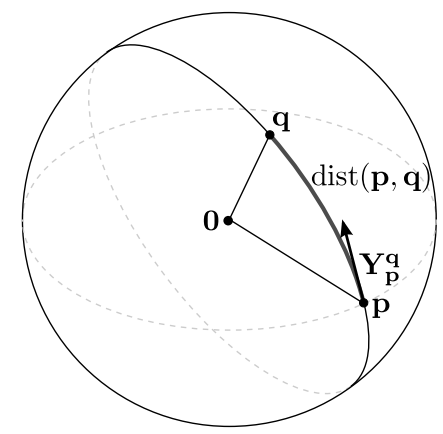

Figure 12: Geodesic on the sphere.

The time-derivative of the geodesic distance between a trajectory $\mathbf{p}=\mathbf{p}(t)$ and a fixed point $\mathbf{q}$ can be expressed in terms of the geodesic direction $\mathbf{Y}_{\mathbf{p}}^{\mathbf{q}}$ :

Lemma 6.1. Consider a trajectory $\mathbf{p}=\mathbf{p}(t)$ on the sphere, such that $\mathbf{p}(t)$ never passes through the fixed points $\mathbf{q}$ or $\mathbf{- q}$. Then

$$
\frac{d}{d t} \operatorname{dist}(\mathbf{p}(t), q)=-\dot{\mathbf{p}} \cdot \mathbf{Y}_{\mathbf{p}}^{\mathbf{q}}
$$

Proof. Differentiating the definition in Equation (24), we have

$$
\begin{aligned}
\frac{d}{d t} \operatorname{dist}(\mathbf{p}(t), \mathbf{q}) & =\frac{d}{d t} \operatorname{acos} \mathbf{p} \cdot \mathbf{q} \\
& =-\frac{\dot{\mathbf{p}} \cdot \mathbf{q}}{\sqrt{1-(\mathbf{p} \cdot \mathbf{q})^{2}}}=-\frac{\dot{\mathbf{p}} \cdot(\mathbf{q}-(\mathbf{q} \cdot \mathbf{p}) \mathbf{p})}{\|\mathbf{p} \times \mathbf{q}\|}=-\dot{\mathbf{p}} \cdot \mathbf{Y}_{\mathbf{p}}^{\mathbf{q}}
\end{aligned}
$$

Let $\mathrm{R}$ be the rotation about $\mathbf{p} \times \mathbf{q}$ which maps $\mathbf{p}$ to $\mathbf{q}$. We will now extend the previous lemma to cover the time-derivative of the geodesic distance between two trajectories $\mathbf{p}=\mathbf{p}(t)$ and $\mathbf{q}=\mathbf{q}(t)$.

Lemma 6.2. Consider a trajectory $\mathbf{p}=\mathbf{p}(t)$ on the sphere, such that $\mathbf{p}(t) \neq \pm \mathbf{q}(t)$ for all $t>0$. Then

$$
\frac{d}{d t} \operatorname{dist}(\mathbf{p}(t), \mathbf{q}(t))=-\left(\dot{\mathbf{p}}-\mathrm{R}^{T} \dot{\mathbf{q}}\right) \cdot \mathbf{Y}_{\mathbf{p}}^{\mathbf{q}}
$$

Proof. By differentiating first with respect to $\mathbf{p}$ and then with respect to $\mathbf{q}$, we have

$$
\frac{d}{d t} \operatorname{dist}(\mathbf{p}, \mathbf{q})=-\dot{\mathbf{p}} \cdot \mathbf{Y}_{\mathbf{p}}^{\mathbf{q}}-\dot{\mathbf{q}} \cdot \mathbf{Y}_{\mathbf{q}}^{\mathbf{p}} .
$$

Since $\mathbf{R} \mathbf{Y}_{\mathbf{p}}^{\mathbf{q}}=-\mathbf{Y}_{\mathbf{q}}^{\mathbf{p}}$, we can simplify the previous expression to

$$
\frac{d}{d t} \operatorname{dist}(\mathbf{p}, \mathbf{q})=-\left(\dot{\mathbf{p}}-\mathrm{R}^{T} \dot{\mathbf{q}}\right) \cdot \mathbf{Y}_{\mathbf{p}}^{\mathbf{q}}
$$




\section{References}

[1] American Wind Energy Association. U.S. Wind Industry Annual Market Report, 2009.

[2] J. D. Anderson. Fundamentals of Aerodynamics. McGraw-Hill Higher Education, New York, 3rd edition, 2001.

[3] K. J. Astrom and B. Wittenmark. Adaptive Control. Addison-Wesley Longman Publishing Co., Inc., 2nd edition, 1994.

[4] T. R. Beal. Digital simulation of atmospheric turbulence for Dryden and von Karman models. Journal of Guidance, Control, and Dynamics, 16(1):132-138, 1993.

[5] J. Breukels. An Engineering Methodology for Kite Design. PhD thesis, Delft University of Technology, Delft, 2011.

[6] F. Bullo, R. M. Murray, and A. Sarti. Control on the sphere and reduced attitude stabilization. Technical report, California Institute of Technology, Pasadena, California, 1995.

[7] S. G. C. de Groot, J. Breukels, R. Schmehl, and W. J. Ockels. Multibody system reduction for derivation of a flight dynamics model for real-time control of kites. Submitted to AIAA Journal of Guidance, Control and Dynamics, 2010.

[8] M. M. Diehl. Real-Time Optimization for Large Scale Nonlinear Processes. PhD thesis, Ruprecht-Karls-Universität, Heidelberg, 2001.

[9] L. Fagiano. Control of Tethered Airfoils for High-Altitude Wind Energy Generation. PhD thesis, Politecnico di Torino, Torino, 2009.

[10] J. Farrell, M. Polycarpou, and M. Sharma. Adaptive backstepping with magnitude, rate, and bandwidth constraints: Aircraft longitude control. In American Control Conference, volume 5, pages 3898-3904, Denver, Colorado, 2003.

[11] A. Gray. Modern Differential Geometry of Curves and Surfaces with Mathematica. CRC-Press, Boca Raton, Florida, 2nd edition, 1997.

[12] B. Houska. Robustness and stability optimization of open-loop controlled power generating kites. Master's thesis, Ruprecht-Karls-Universität, Heidelberg, 2007.

[13] B. Houska and M. M. Diehl. Optimal control for power generating kites. In 9th European Control Conference, pages 3560-3567, Kos, Greece, 2007.

[14] O. Härkegård and S. T. Glad. Flight control design using backstepping. Division of Automatic Control, Department of Electrical Engineering, Linköpings universitet, Linköping, 2001.

[15] A. Ilzhöfer, B. Houska, and M. M. Diehl. Nonlinear MPC of kites under varying wind conditions for a new class of large scale wind power generators. International Journal of Robust and Nonlinear Control, 17(17):1590-1599, 2007. 
[16] H. K. Khalil. Nonlinear Systems. Prentice Hall, Upper Saddle River, 3rd edition, 2001.

[17] M. Krstic, I. Kanellakopoulos, and P. V. Kokotovic. Nonlinear and adaptive control design. John Wiley \& Sons, New York, 1995.

[18] B. Lansdorp, R. Ruiterkamp, P. Williams, and W. J. Ockels. Long-term laddermill modeling for site selection. In AIAA 2008-6690, 2008.

[19] M. L. Loyd. Crosswind kite power. Journal of Energy, 4(3):106-111, 1980.

[20] J. P. Meijaard, W. J. Ockels, and A. L. Schwab. Modelling of the dynamic behaviour of a Laddermill, a novel concept to exploit wind energy. In $3 r d$ International Symposium on Cable Dynamics, Trondheim, 1999.

[21] W. J. Ockels. Laddermill, a novel concept to exploit the energy in the airspace. Aircraft Design, 4(2-3):81-97, 2001.

[22] R. E. Sheldahl and P. C. Klimas. Aerodynamic characteristics of seven symmetrical airfoil sections through 180-degree angle of attack for use in aerodynamic analysis of vertical axis wind turbines. Technical report, Sandia National Laboratories, Albuquerque, NM, 1981.

[23] S. Sieberling, Q. P. Chu, and J. A. Mulder. Robust flight control using incremental nonlinear dynamic inversion and angular acceleration prediction. Journal of Guidance, Control, and Dynamics, 33(6):1732-1742, 2010.

[24] L. Sonneveldt, Q. P. Chu, and J. A. Mulder. Nonlinear flight control design using constrained adaptive backstepping. Journal of Guidance, Control, and Dynamics, 30(2):322-336, 2007.

[25] P. Williams, B. Lansdorp, and W. J. Ockels. Flexible tethered kite with moveable attachment points, part I: Dynamics and control. In AIAA Atmospheric Flight Mechanics Conference and Exhibit, Hilton Head, South Carolina, 2007.

[26] P. Williams, B. Lansdorp, and W. J. Ockels. Nonlinear control and estimation of a tethered kite in changing wind conditions. Journal of Guidance, Control and Dynamics, 31(3):793-799, 2008. 